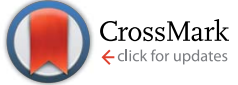

Cite this: J. Mater. Chem. A, 2015, 3, 18963

Received 2nd July 2015

Accepted 10th August 2015

DOI: $10.1039 / c 5 t a 04930 j$

www.rsc.org/MaterialsA

\section{A facile strategy for the fabrication of a bioinspired hydrophilic-superhydrophobic patterned surface for highly efficient fog-harvesting $\dagger$}

\author{
Yuchao Wang, ${ }^{a}$ Lianbin Zhang, ${ }^{a}$ Jinbo $\mathrm{Wu}^{\mathrm{a}}$ Mohamed Nejib Hedhilib \\ and Peng Wang*a
}

Fog water collection represents a meaningful effort in places where regular water sources, including surface water and ground water, are scarce. Inspired by the amazing fog water collection capability of the Stenocara beetles in the Namib Desert and based on the recent work in biomimetic water collection, this work reports a facile, easy-to-operate, and low-cost method for the fabrication of a hydrophilic-superhydrophobic patterned hybrid surface towards highly efficient fog water collection. The essence of the method is incorporating a (super)hydrophobically modified metal-based gauze onto the surface of a hydrophilic polystyrene (PS) flat sheet by a simple lab oven-based thermal pressing procedure. The produced hybrid patterned surfaces consisted of PS patches sitting within the holes of the metal gauzes. The method allows for easy control over the pattern's dimensions (e.g., patch size) by varying the gauze mesh size and the thermal pressing temperature, which is then translated into the easy optimization of the ultimate fog water collection efficiency. Given the low-cost and wide availability of both PS and the metal gauze, this method has great potential for scaling-up. The results showed that the hydrophilicsuperhydrophobic patterned hybrid surfaces with a similar pattern size to the Stenocara beetles's back pattern produced a significantly higher fog collection efficiency than the uniformly (super)hydrophilic or (super)hydrophobic surfaces. This work contributes to the general effort in fabricating mixed wettability patterned surfaces for atmospheric water collection for direct use.

\section{Introduction}

Atmospheric water, especially fog, represents a significant but largely untapped fresh water source, especially in semi-arid, desert regions, land-scarce regions as well as countries with high economic activities. ${ }^{\mathbf{1 , 2}}$ In the past decade, research attention has been focused on learning from nature and on biomimetically imitating the ways of capturing atmospheric water by some natural creatures, among which the Stenocara beetles, which survive in the Namib Desert, have inspired many scientists. ${ }^{3-6}$ It has been revealed that the great fog water collection capability of the beetles results from the special structure on their back, which consists of an array of hydrophilic bumps distributed on a superhydrophobic background. ${ }^{7,8}$ It is generally believed that the hydrophilic bumps are conducive to fog

${ }^{a}$ Water Desalination and Reuse Center, Division of Biological and Environmental Science and Engineering, King Abdullah University of Science and Technology, Thuwal 23955-6900, Saudi Arabia.E-mail: peng.wang@kaust.edu.sa

${ }^{b}$ Imaging and Characterization Laboratory, King Abdullah University of Science and Technology, Thuwal 23955-6900, Saudi Arabia

$\dagger$ Electronic supplementary information (ESI) available: Additional SEM details of the hybrid surfaces prepared at different temperature, and the water contact angles of the hybrid surfaces. See DOI: 10.1039/c5ta04930j droplet capture and then coalescence while the hydrophobic background helps to clear the water droplets from the surface once they reach a certain size. ${ }^{9,10}$

For producing such hydrophilic-hydrophobic patterned surfaces, three major strategies are generally utilized: (i) randomly dispersing hydrophilic glass spheres on a hydrophobic waxy substance; ${ }^{3}$ (ii) the mask-based lithograph method $;^{11-13}$ and (iii) direct-patterning by inkjet printing. ${ }^{14-17}$ Strategies (ii) and (iii) are capable of precisely producing predesigned patterns, while strategy (i) is incapable of this. However, the mask-based strategy consists of mask preparation, pattern transfer and pattern wettability adjustment, which is lengthy and has multiple steps. While the inkjet printing method, although it is a one-step procedure, demands a special printer to print well-controlled patterns. Thus, a facile, simple and easy-to-operate method that is able to produce a stable hydrophilic-hydrophobic patterned surface with low-cost is still highly sought after.

On such a patterned surface, there are at least two portions with different wettabilities (hydrophilic versus hydrophobic), so one possibility would be to simply press together materials with different wettabilities so that they both appear to make a composite surface with pattern dimensions suitable for fog collection. Following this new idea, the following requirements 
have to be met: (i) the two materials possess different wettabilities or can undergo wettability modification, and (ii) one material has to be porous with a suitable pore size and the other has to be made flexible so the two can come together by a simple treatment to build a composite surface. This way, the porous material literally serves as a mask in producing the patterned surface and the flexible one is then considered as the molding material in the same process.

Gauzes are commonly seen in our daily life, such as mosquito screens, kitchen strainers and colanders. They are made of polymeric materials (e.g., polypropylene), metals (e.g., copper, nickel, iron, titanium), or stainless steel and can have different mesh sizes, depending on their intended purposes. ${ }^{\mathbf{1 8 - 2 0}}$ Among all of these gauze materials, the metal gauzes attract our attention as a candidate for the mask material in producing the patterned surface, due to their suitability for surface chemical modification, their mechanical strength and long-term stability. ${ }^{21,22}$ On the other hand, polystyrene (PS) is a kind of lowcost and widely used commercial wettable polymer with a contact angle that is generally less than $90^{\circ}$ and it is thus considered a hydrophilic material. ${ }^{23}$ Additionally, PS has a relatively low processing temperature, with a glass transition temperature around $80-100{ }^{\circ} \mathrm{C} .{ }^{24}$ With the metal gauze being a mask material, PS can then be a suitable choice as a molding material to make the composite surface. ${ }^{25}$

Herein, we develop a simple and controllable technique to fabricate a well-patterned composite surface for fog harvesting by combining two commonly available materials: copper gauzes and PS plane sheets. The copper gauzes were modified to be superhydrophobic, which was then combined with the hydrophilic PS plane sheet directly by thermal-pressing to successfully achieve a hydrophilic PS patched patterned superhydrophobic copper mesh surface. Our method is low-cost and easy-to-operate and allows for easy control of the mesh size and thus pattern size. The produced surface exhibits excellent fog collection efficiency, showing a much better performance than uniformly hydrophilic and superhydrophobic surfaces. This work contributes to general effort in fabricating mixed wettability patterned surfaces for atmospheric water collection for direct use.

\section{Experimental}

\section{Materials}

Copper gauzes with different mesh sizes (50 mesh with a $0.23 \mathrm{~mm}$ wire diameter $/ 270 \mu \mathrm{m} \times 270 \mu \mathrm{m}$ pore size, 60 mesh with a $0.19 \mathrm{~mm}$ wire diameter $/ 250 \mu \mathrm{m} \times 250 \mu \mathrm{m}$ pore size, 80 mesh with a $0.14 \mathrm{~mm}$ wire diameter $/ 180 \mu \mathrm{m} \times 180 \mu \mathrm{m}$ pore size, and 100 mesh with a $0.11 \mathrm{~mm}$ wire diameter/145 $\mu \mathrm{m} \times$ $145 \mu \mathrm{m}$ pore size) were purchased from Alfa Aesar (Karlsruhe, Germany). Copper foil with a thickness of $1.0 \mathrm{~mm}$, hydrochloric acid ( $\mathrm{HCl}, 37 \%)$, absolute ethanol $(\geq 99.8 \%$ ) and $1 \mathrm{H}, 1 \mathrm{H}, 2 \mathrm{H}, 2 \mathrm{H}$ perfluorodecanethiol (PFDT, 97\%) were obtained from SigmaAldrich (St. Louis, MO, USA). A polystyrene (PS) plane sheet was purchased from Thermo Fisher Scientific (Waltham, MA, USA). As shown in Fig. S1 in ESI, $\dagger$ the water contact angle (CA) of the PS sheet was about $76^{\circ}$, and so defined as a hydrophilic surface.
All chemicals were used as received. De-ionized (DI) water purified in a Milli-Q (Millipore, Billericay, MA, USA) system was used in all experiments.

\section{The preparation of the superhydrophobic surface on copper gauzes}

The preparation of the hydrophilic-superhydrophobic patterned surface is schematically shown in Scheme 1. The copper gauzes were first immersed in a $4 \mathrm{M} \mathrm{HCl}$ aqueous solution for a few seconds, and then washed with copious amounts of ethanol and deionized water. The pre-cleaned copper gauzes were calcined at $400{ }^{\circ} \mathrm{C}$ for $3 \mathrm{~h}$ in an oven to form surface copper oxide nanostructures. To convert the hydrophilic gauzes into hydrophobic ones, the gauzes with a black copper oxide layer were immersed into a $1.0 \% \mathrm{v} / \mathrm{v}$ PFDT ethanol solution for 20 minutes, followed by washing with ethanol and drying by nitrogen flow. The resultant samples were denoted as CuO- $x$-PFDT, with $x$ being the gauze mesh size and PFDT being the fluorine compound modification. While, the copper gauzes that weren't calcined (denoted as Cu- $x$-PFDT) and thus without the metal oxide surface layer, were hydrophobically modified with the same procedure that was done to form CuO- $x$-PFDT and so a uniformly superhydrophobic surface was fabricated on the copper foil, which was denoted as CuO-PFDT.

\section{The preparation of the composite samples}

A piece of superhydrophobic CuO- $x$-PFDT with a dimension of $2.5 \mathrm{~cm} \times 2.5 \mathrm{~cm}$ was placed on a same sized PS sheet and a fixed pressure was applied on top to keep the two pieces of materials together, which were then transferred together and heated in an

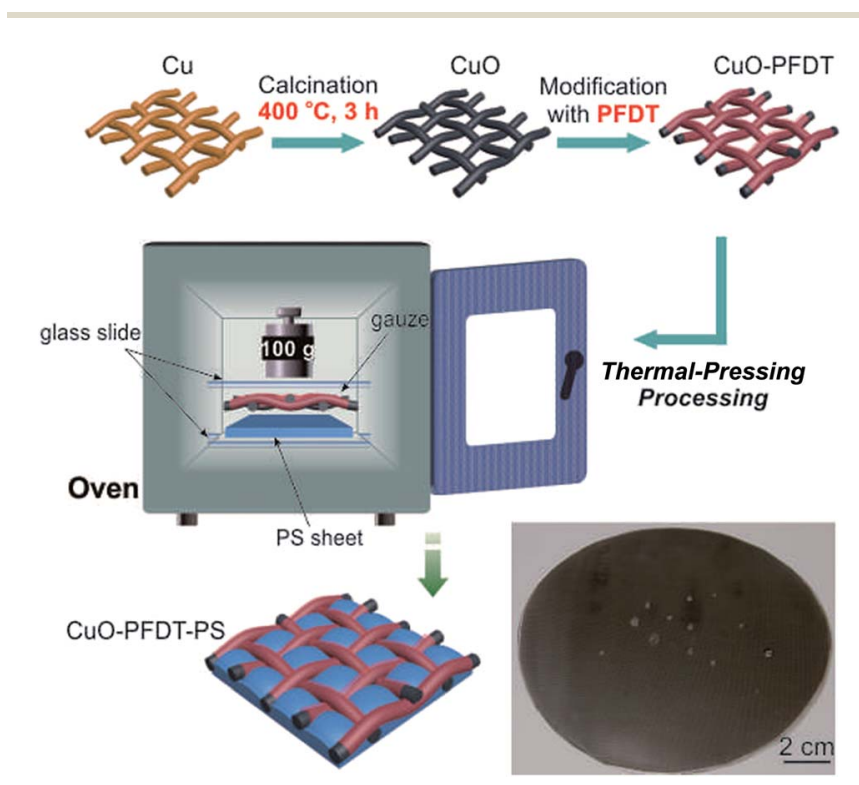

Scheme 1 The preparation procedure for the hydrophilic-superhydrophobic patterned composite surface: (i) calcination of the copper gauze to form the oxide surface coating layer; (ii) hydrophobic modification of the oxide coated copper gauze with PFDT; (iii) thermal-pressing of the PFDT modified gauze and the PS sheet to form the composite surface with patterned wettability. The insert image presents some water droplets sitting on a composite sample surface. 
oven at a pre-defined temperature (i.e., 120, 130, 140, 150, $160{ }^{\circ} \mathrm{C}$ ) for 3 hours (Scheme 1). The resultant composite samples were indicated as CuO- $x$-PFDT-PS- $y$, where $x$ represented the mesh size of the copper gauze and $y$ represented the thermal-treatment temperature. For comparison, the $\mathrm{Cu}-x$-PFDT sample was thermally pressed with PS by the same method and the corresponding composite sample was denoted as Cu- $x$-PFDT-PS- $y$.

\section{Material characterization}

Scanning electron microscopy (SEM) images were taken with an FEI Quanta 600 scanning electron microscope (FEI Co., Hillsboro, OR, USA). The contact angle and sliding angle data were obtained on a commercial contact angle system (OCA 35, Data-Physics, Filderstadt, Germany) at ambient temperature using a $5 \mu \mathrm{L}$ droplet as the indicator. The crystalline structure of the samples was analyzed by X-ray diffraction (XRD) (Bruker D8 Discover diffractometer). X-ray photoelectron spectroscopy (XPS) measurements were made with an AXISNOVA instrument (Kratos Analytical Ltd, Manchester, UK) using a monochromatic Al K $\propto$ X-ray source $(1486.6 \mathrm{eV})$. Digital photographs were captured using a Canon EOS 7D.

\section{Fog-harvesting measurements}

A homemade test system was built in our lab to evaluate the fogharvesting performance of our samples, which is schematically presented in Fig. 6a. The as-prepared samples $(2.5 \mathrm{~cm} \times 2.5 \mathrm{~cm}$ in size) were fixed on a holder at ambient conditions. The sample was held so that its surface was perpendicular to the horizontal plane. A simulated flow of fog (about $12 \mathrm{~cm} \mathrm{~s}^{-1}$ ) was generated by a commercial humidifier and captured by the vertically placed sample surfaces. The distance between the fog generator and the sample was kept at $7 \mathrm{~cm}$. The duration of one cycle measurement is four hours. The temperature and relative humidity around the samples were $22{ }^{\circ} \mathrm{C}$ and 90-95\%, respectively. Water droplets collected by the surfaces were drained by gravity into a container placed on top of a digital balance.

\section{Results and discussions}

As shown in Scheme 1, a pre-cleaned copper gauze was first calcined to form a copper oxide $(\mathrm{CuO})$ coating layer and the CuO-coated gauze was then modified by PFDT to render a superhydrophobic mesh surface. The PFDT-modified gauze was finally thermally pressed together with a hydrophilic PS sheet (contact angle $76^{\circ}$, Fig. S1 in ESI $\dagger$ ) to give rise to a hydrophilically-patterned superhydrophobic hybrid surface. Due to the nature of the method, the produced composite surface assumed a three-dimensional concaving surface structure at the microscale, with the hydrophilic PS patches sitting within the mesh holes and serving as the bottoms of the concaves. The image at the bottom of Scheme 1 is a digital photo of a hybrid circular material with a diameter of $12 \mathrm{~cm}$ produced by the current method, demonstrating its scalability. To facilitate discussion, from this point on, the samples prepared on 50\# mesh gauzes were chosen for the focused discussion unless otherwise noted.
Fig. 1 presents SEM images of the samples with a 50\# mesh size at different stages of the preparation. Each thread of the pre-cleaned copper gauze showed a generally smooth surface with some mechanical scratches in the micro-scale (Fig. 1a and b). While after calcination at $400{ }^{\circ} \mathrm{C}$, the gauze thread surface was fully covered with a uniform oxide layer formed by stacks of particles in the tens of nanometers and copper oxide nanowires with lengths of 2.5-9.5 $\mu \mathrm{m}$ growing from the gaps among the oxides particles (Fig. 1c-e), which significantly enhanced the surface roughness at the nanoscale. Such an oxide structure on the copper gauze surface fitted well with the results reported by Xia's group. ${ }^{26}$ Since the chemical composition is one important factor for the wettability of a solid surface, XRD and XPS were employed to investigate the crystal structure and the chemical composition of the gauze samples. As shown in the XRD data, except for the strong peaks at $2 \theta=43.3^{\circ}, 50.5^{\circ}$ and $74.2^{\circ}$ which related to pure copper, several other diffraction peaks emerged, which can be ascribed to the formation of $\mathrm{CuO}$ and $\mathrm{Cu}_{2} \mathrm{O}$ (Fig. 2a and b). It indicated that a $\mathrm{CuO}$ layer with some $\mathrm{Cu}_{2} \mathrm{O}$ minor phase was formed on the surface of the $\mathrm{Cu}$ gauze after the calcination. Fig. 1f through to Fig. 1h present the SEM images of the gauze surface after the PFDT modification. Clearly the surface $\mathrm{CuO}$ nanowire structures persisted after the hydrophobic modification and after the thermal pressing step (Fig. S2 in ESI $\dagger$ ), indicating the stability of the nanostructures of the $\mathrm{CuO}$ layer. From the SEM images under different magnifications of the pre-cleaned gauze before (Fig. 1a and b) and after the PFDT modification (Fig. 1i and j), one can see that both surfaces were similarly smooth, implying that the PFDT modification method in this work did not significantly affect the surface morphology and roughness of the gauzes.

Fig. $2 \mathrm{c}$ and $\mathrm{d}$ present the full XPS spectra of the original $\mathrm{CuO}$ gauze and the PFDT modified $\mathrm{CuO}$ gauze. A strong $\mathrm{O}$ 1s signal peak at $529.8 \mathrm{eV}$ on the $\mathrm{CuO}$ sample indicates the formation of the copper oxide layer. In the XPS spectrum of CuO-PFDT, new bands at $164.1 \mathrm{eV}$ and $684.9 \mathrm{eV}$ were revealed, which indicates $\mathrm{S}$ $2 \mathrm{p}$ and $\mathrm{F} 1 \mathrm{~s}$ respectively from PFDT. ${ }^{27,28}$ The atomic content of $\mathrm{F}$ was estimated to be as high as $44.3 \%$. This result confirms the successful PFDT functionalization of the copper gauze. PFDT is a widely used surface hydrophobic modification reagent and it has been proven that it can easily react with many metals and metal oxides and form covalent bonds. ${ }^{29-32}$ More details could be obtained from the high-resolution photoelectron spectra of $\mathrm{Cu} 2 \mathrm{p}$ in Fig. 2e and f. For the CuO-50-PFDT surface, two different $\mathrm{Cu} 2$ p3/2 peaks were observed. One peak at $933.8 \mathrm{eV}$ corresponded to $\mathrm{Cu}$ in the copper oxides. The other peak at 931.4 eV occurred at the binding energy of $\mathrm{Cu}$ in $\mathrm{Cu}-\mathrm{S}$. It indicated that there was a covalent-like bonding between $\mathrm{Cu}$ (in the surface) and S (in PFDT). ${ }^{33,34}$ The covalent bonding between PFDT and $\mathrm{Cu}-\mathrm{S}$ makes sure that the obtained surface is a stable superhydrophobic surface. By carefully weighing the samples before and after the PFDT modification, a slight weight increase of $0.026 \mathrm{wt} \%$ was recorded due to the PFDT surface grafting.

As shown in Fig. 3a and Table 1, the pre-cleaned copper gauze before calcination showed hydrophobicity with a static water contact angle of about $109^{\circ} .^{35-37}$ After the calcination, the uniform $\mathrm{CuO}$ coating layer with the nanowire structure 


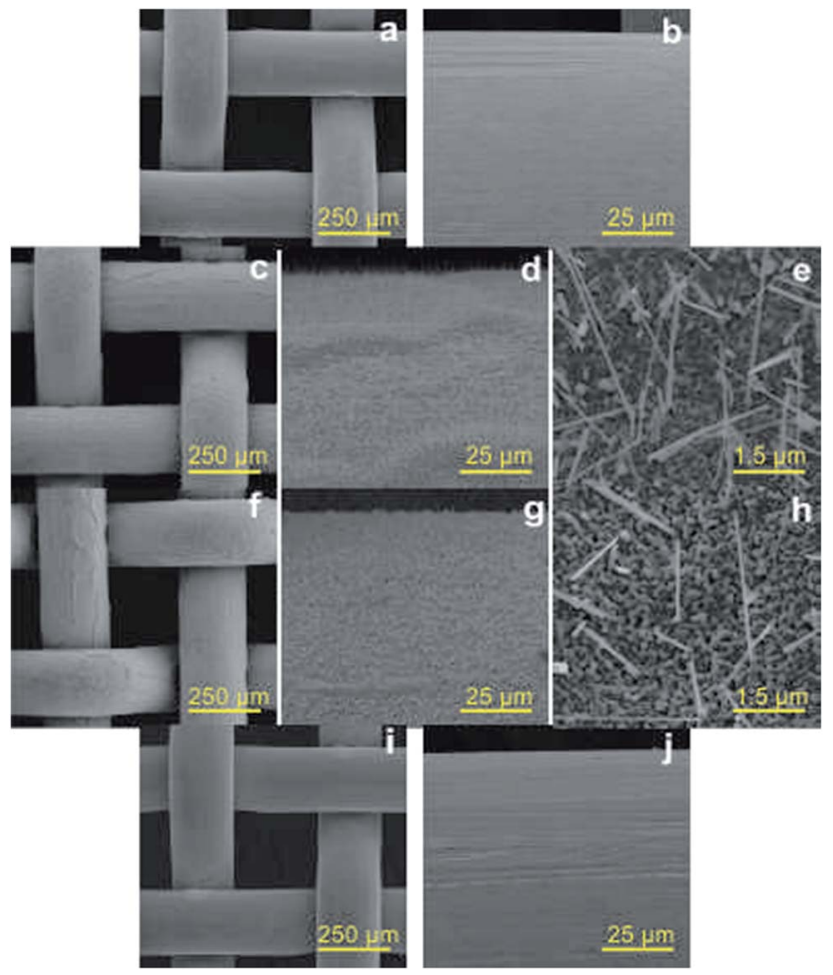

Fig. 1 SEM images of the samples prepared from the 50\# gauzes at different preparation stages: $\mathrm{Cu}(\mathrm{a}$ and $\mathrm{b}), \mathrm{CuO}(\mathrm{c}-\mathrm{e}), \mathrm{CuO}-\mathrm{PFDT}(\mathrm{f}-\mathrm{h})$ and Cu-PFDT (i and j).
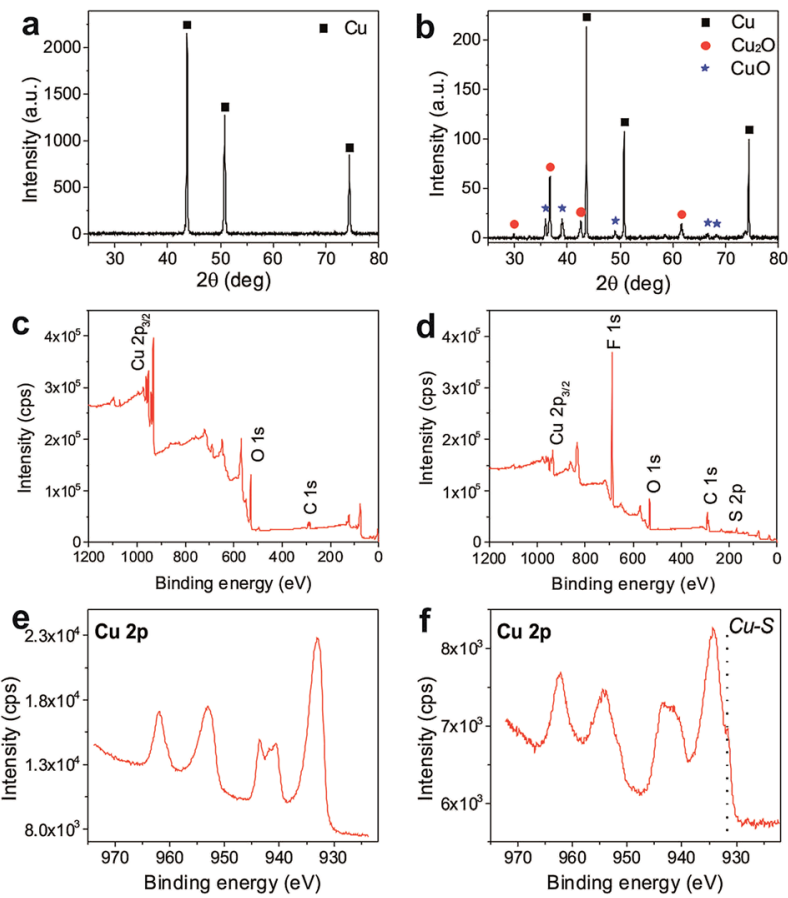

Fig. 2 XRD patterns of the raw Cu gauze (50\# mesh) (a) and CuO-50 (b), XPS spectra of CuO-50 (c), CuO-50-PFDT (d), and the $\mathrm{Cu} 2 \mathrm{p}$ spectra of CuO-50 (e) and CuO-50-PFDT (f). drastically turned a hydrophobic surface into a superhydrophilic one with a water contact angle of $4^{\circ} \cdot{ }^{38}$ A static water contact angle $\geq 150^{\circ}$ and a water sliding angle $\leq 10^{\circ}$ were obtained on the surface of the PFDT modified CuO gauzes, indicating real superhydrophobic surfaces with a Cassie's wetting type. Beside the hydrophobic PFDT functional groups on the surface, the surface wettability of CuO-PDFT can be ascribed to the inherent microscale mesh structure of the gauzes, and the surface $\mathrm{CuO}$ nanostructures on each thread of the gauzes. However, the PFDT modified $\mathrm{Cu}$ gauze (Cu-PFDT), namely the $\mathrm{Cu}$ gauze without calcination at $400{ }^{\circ} \mathrm{C}$, exhibited a highly hydrophobic property with a water contact angle of $147^{\circ}$ (Fig. 3d) but it also showed high surface adhesion, suggesting that the surface had difficulty in removing water droplets from itself. The high surface adhesion of Cu-PDFT may be due to the small surface roughness caused by the lack of micro-structures on its surface. ${ }^{39}$ Such a hydrophobic surface with a high sliding angle would not be conducive for an efficient fog water collection system in which a balance must be struck between fog droplet capture and the clearance of the droplets off the surface. ${ }^{40}$ The rational comparison clearly demonstrates the necessity of the surface oxide layer by calcination, which gives rise to the ultimate superhydrophobic gauze after PFDT modification with a Cassie wetting behavior. After the thermal pressing with PS, the wettability of the resulted hybrid patterned surface was similar to the gauze surface before the pressing (Fig. S3 in ESI $\dagger$ ).

One of the attractive features of this method is that the pattern size can be easily and conveniently controlled by choosing a gauze with a different mesh number. Fig. 4 presents

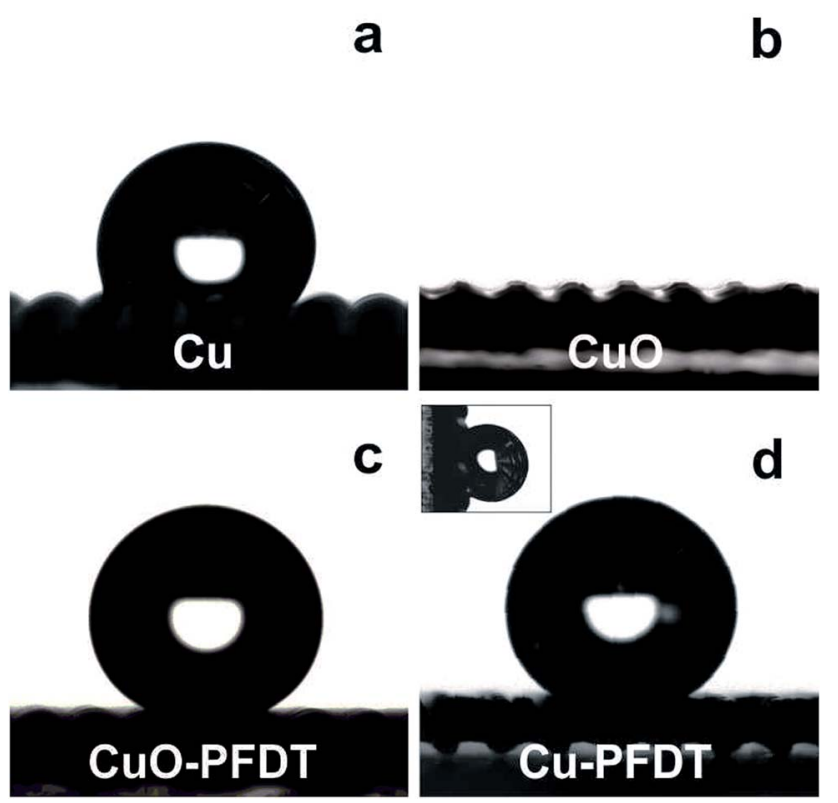

Fig. 3 The CA measurement images of the sample prepared from $50 \#$ gauze at different preparation stages, including (a) $\mathrm{Cu}$, (b) $\mathrm{CuO}$, (c) $\mathrm{CuO}-\mathrm{PFDT}$ and (d) Cu-PFDT. The inset image is the water contact angle of $\mathrm{Cu}$-PFDT with tilt angles of $90^{\circ}$. All of the water droplet volumes in these images were $5 \mu \mathrm{L}$. 
Table 1 The water contact angle and sliding angle data of the 50\# gauze samples

\begin{tabular}{llllll}
\hline & $\mathrm{Cu}^{a}$ & $\mathrm{CuO}$ & $\mathrm{CuO}-\mathrm{PFDT}$ & $\mathrm{Cu}-\mathrm{PFDT}$ & $\mathrm{PS}^{c}$ \\
\hline Water contact angle & $109 \pm 8^{\circ}$ & $<4^{\circ}$ & $161 \pm 3^{\circ}$ & $147 \pm 3^{\circ}$ & $76 \pm 4^{\circ}$ \\
Water sliding angle & $\mathrm{NA}^{b}$ & & $8 \pm 1^{\circ}$ & $\mathrm{NA}^{b}$ & $\mathrm{NA}^{b}$
\end{tabular}

${ }^{a}$ All of the water contact angle results are based on the gauze with 50\# mesh number. ${ }^{b}$ Liquid droplets were pinned on the surfaces with high adhesion, and no sliding behavior was observed during tilting of the substrates. ${ }^{c}$ The PS sheet was obtained from Thermo Fisher Scientific.

the SEM images of the hybrid surfaces composed of PS and PFDT modified $\mathrm{CuO}$ gauzes with different mesh numbers. As can be clearly seen, uniform patterns of PS with different dimensions were successfully produced. For example, the PS domain's area could be adjusted from $7.3 \times 10^{4} \mu \mathrm{m}^{2}$ to $2.1 \times$ $10^{4} \mu \mathrm{m}^{2}$ when the mesh number increased from $50 \#$ to $100 \#$ respectively. The versatility in controlling the pattern size allows for easy optimization of the water collection efficiency later.

An added benefit of the thermal pressing in this work is that it permits easy control over the height of the PS patches within the mesh holes of the gauzes. Fig. 5 presents cross-section SEM images of the PS patches with different thermal pressing temperatures to show the variation of the PS patch height as a function of the temperature. For the purpose of clear observation, the superhydrophobic CuO-PFDT gauzes were removed from the PS sheets when taking the SEM images. Due to its low glass transition temperature, the PS sheet is softer and more moldable under higher temperatures, leading to a higher PS patch height under a higher treatment temperature (Fig. 5 and S4 in ESI $\dagger$ ). The PS patch height, a parameter which is generally overlooked, was later found to be a relevant parameter in the fog collection efficiency. ${ }^{41}$

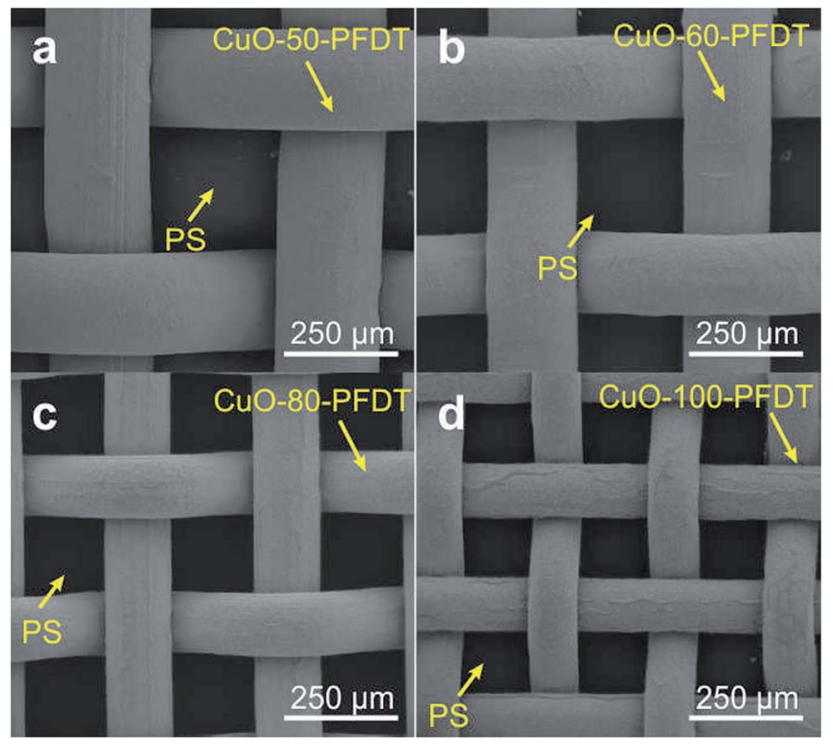

Fig. 4 The top view SEM images of the hybrid surfaces composed of the PS and PFDT modified $\mathrm{CuO}$ gauzes with different mesh numbers (a) 50\#, (b) 60\#, (c) 80\#, (d) 100\#
To verify the performance of the designed hydrophilicsuperhydrophobic patterned composite surfaces, the fog-harvesting efficiencies of these surfaces were then investigated by a homemade fog-harvesting system, which is schematically presented in Fig. 6a. In brief, a commercial humidifier was used to generate a simulated fog flow and the prepared composite sample was held vertically. Fog water was collected onto the patterned composite surface at ambient conditions, drained by gravity and collected in a glass container placed on a digital balance which was connected to a computer. The water collection rates of six samples with different surface wettabilities are listed in Fig. 6b, and the six samples were CuO-50-PFDT-PS-130 (hydrophilic-superhydrophobic patterned surface), Cu-50-PS-130 (hydrophobic gauze without PFDT modification but with PS), CuO-50-PS-130 (superhydrophilic gauze with PS), Cu-50-PFDT-PS130 (highly hydrophobic gauze with a high sliding angle with PS), a flat PS sheet, and superhydrophobic CuO-PFDT foil. The hydrophilic-superhydrophobic hybrid surface on the CuO-50-PFDT-PS-130 exhibited a water collection rate of $159 \mathrm{mg}$ $\mathrm{cm}^{2} \mathrm{~h}^{-1}$, the highest among all of the six samples tested, whereas, in a sharp contrast, the water collection rates on the other five samples were all no better than $68 \mathrm{mg} \mathrm{cm}^{2} \mathrm{~h}^{-1}$. Such a huge difference in the water collection efficiency clearly demonstrates the great benefit of having a hydrophilic-superhydrophobic patterned surface for water collection. ${ }^{\mathbf{4 2 - 4 4}}$

The uniform superhydrophobic CuO-PFDT foil and uniform hydrophilic PS sheet generated water collection rates of 67 and $60 \mathrm{mg} \mathrm{cm}^{2} \mathrm{~h}^{-1}$, respectively, which are both far lower than the CuO-50-PFDT-130 sample with the hydrophilic-superhydrophobic patterned surface. It is generally believed that on a uniform hydrophilic surface, water droplets tend to spread out and form a thin film, which is reluctant to leave the surface

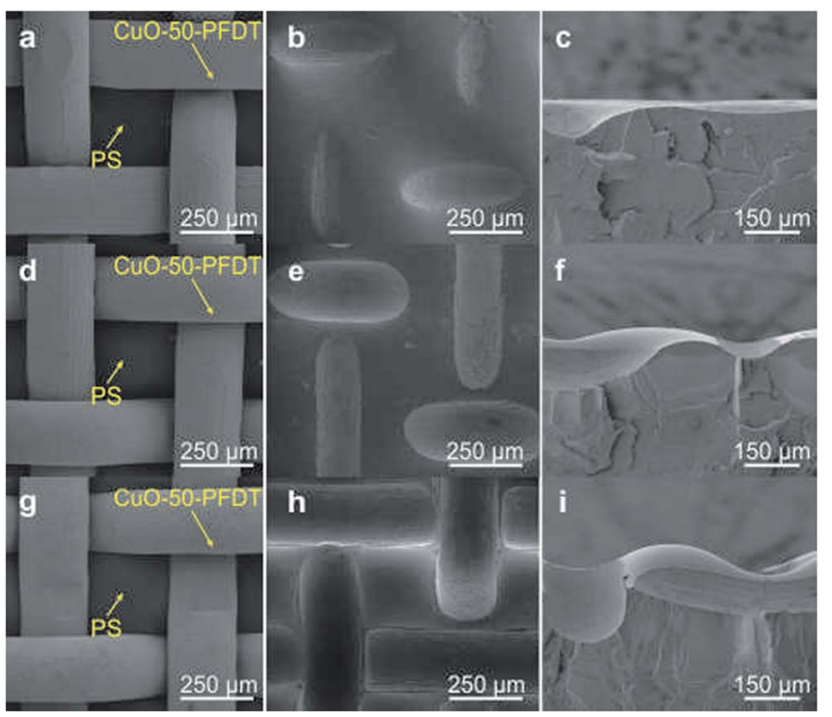

Fig. 5 The SEM images of composite surfaces composed of the PS and PFDT modified $\mathrm{CuO}$ gauzes with $50 \#$ mesh size prepared under different thermal treatment temperature ( $a, d$ and g), and the SEM top view (b, e and $h$ ) and cross-section view ( $c, f$ and i) of the PS surface morphology $\left((a-c)\right.$ at $120^{\circ} \mathrm{C} ;(d-f)$ at $130{ }^{\circ} \mathrm{C} ;(g-i)$ at $\left.140{ }^{\circ} \mathrm{C}\right)$ with the gauzes being removed. 


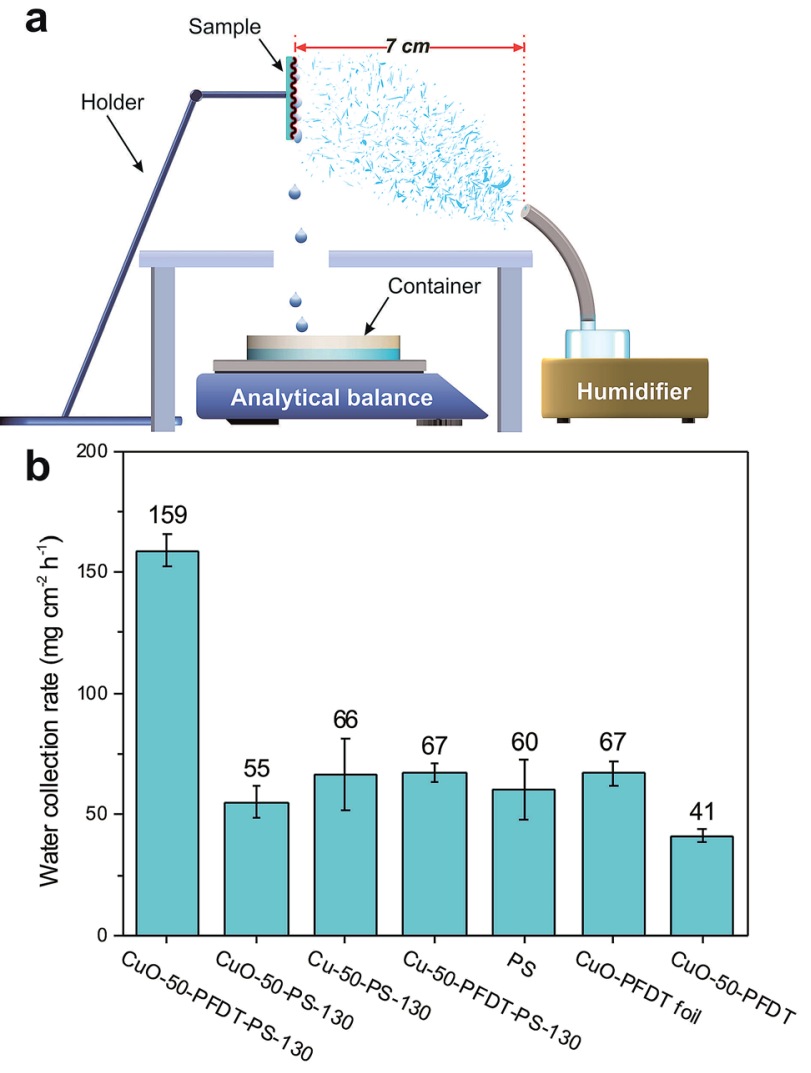

Fig. 6 (a) Schematic illustration of the homemade fog-harvesting system. (b) Water collection rates of the different samples.

while the tendency of small water droplets to grow bigger is inhibited on a uniform (super)hydrophobic surface. ${ }^{15,41,45}$ On the other hand, on the patterned surface, the small water droplets that are captured on the superhydrophobic regions preferentially move toward the hydrophilic regions, driven by the wettability differences, and subsequently coalesce into bigger droplets in these regions. As the droplets in the hydrophilic regions grow beyond a certain threshold, they are removed from the surface by gravity, ${ }^{46}$ which is supported by our real-time observation of water droplet movement and growth on the CuO-50-PFDT-PS-130 surface. Thus, the patterned surface nicely integrates and balances surface water droplet coalescence and droplet removal, which are two competing processes in fog water collection, because droplet coalescence requires hydrophilicity, whereas droplet removal benefits from superhydrophobicity on the patterned surface.

The water collection rates of the other composite samples (Cu-50-PS-130, CuO-50-PS-130 and Cu-50-PFDT-PS-130) were $55 \mathrm{mg} \mathrm{cm}^{2} \mathrm{~h}^{-1}, 66 \mathrm{mg} \mathrm{cm}^{2} \mathrm{~h}^{-1}$ and $67 \mathrm{mg} \mathrm{cm}^{2} \mathrm{~h}^{-1}$, respectively. The main difference among these three samples was the degree of hydrophobicity of the gauzes, demonstrating again the necessity of both the surface $\mathrm{CuO}$ coating layer and PFDT modification for the optimized water collection performance. The water collection rates of the composite surfaces on the gauzes with different mesh numbers (i.e., 50\#, 60\#, 80\# and $100 \#)$ are shown in Fig. 7a. The CuO-50-PFDT-PS-130 with a patterned PS patch size of about $7.3 \times 10^{4} \mu \mathrm{m}^{2}$ and a separation
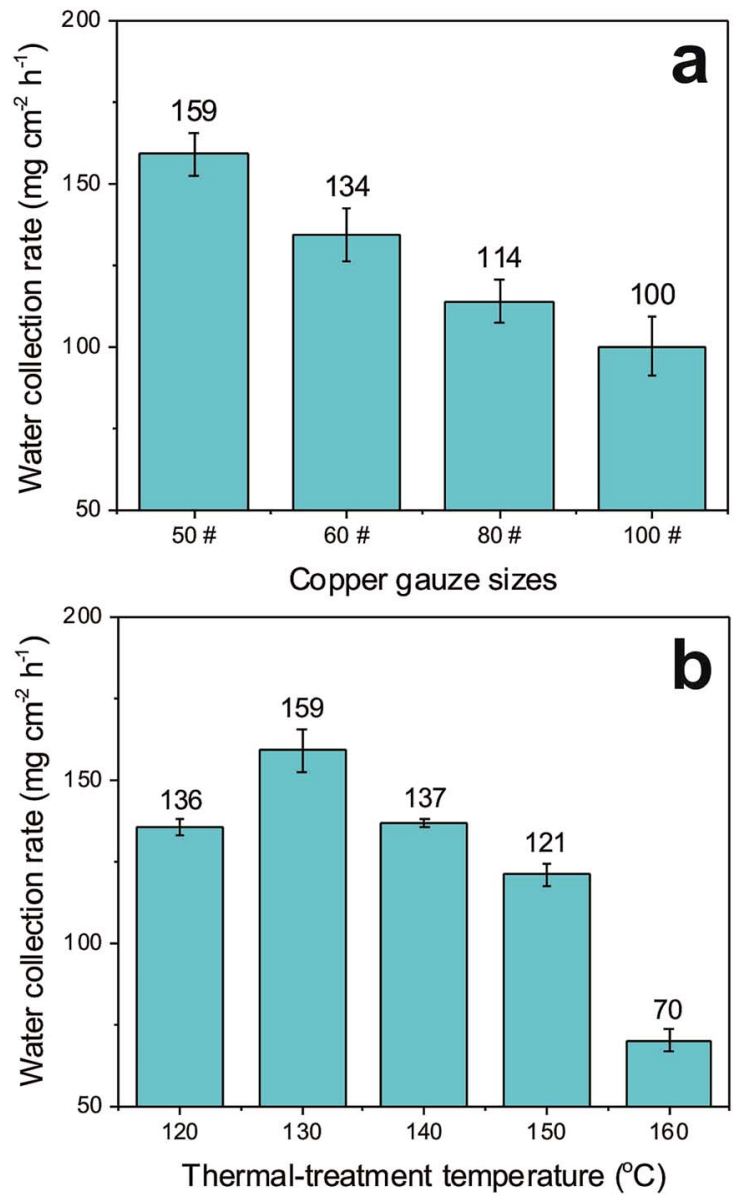

Fig. 7 (a) Water collection rates as a function of mesh number; (b) the comparison of the water collection efficiency by the samples prepared at different thermal treatment temperatures.

distance of about $222 \mu \mathrm{m}$ exhibited the highest efficiency among all of the samples, which, not surprisingly, was similar to the patch dimensions on the back of the Stenocara beetles. ${ }^{15}$ Fig. 7b compares the fog-harvesting performance of the composite surfaces with different concave heights prepared on the 50\# gauzes by using different thermal treatment temperatures (Fig. S4 in ESI $\dagger$ ), among which, the CuO-50-PFDT-PS-130 possessed the best performance. The result shows that the height of the PS hydrophilic patches within the mesh holes is not a trivial factor and the mechanism behind the relationship in Fig. $7 \mathrm{~b}$ is currently under investigation in our group.

\section{Conclusions}

In summary, we developed a facile, easy-to-operate, and lowcost method for the fabrication of hydrophilic-superhydrophobic patterned hybrid surfaces, which provide excellent performance in fog water collection. The method is based on the thermal pressing of a hydrophilic PS sheet with a superhydrophobic gauze and the convenient control over the pattern size and the pattern height in the method allows for easy optimization of the fog water collection efficiency. We believe that 
the current method has great practical value in large-scale applications due to its high efficiency and scalability.

\section{Acknowledgements}

The authors are grateful to KAUST CRG-2 for funding.

\section{References}

1 Q. Schiermeier, Nature, 2014, 505, 10-11.

2 M. M. Pendergast and E. M. V. Hoek, Energy Environ. Sci., 2011, 4, 1946-1971.

3 A. R. Parker and C. R. Lawrence, Nature, 2001, 414, 33-34.

4 J. Ju, H. Bai, Y. Zheng, T. Zhao, R. Fang and L. Jiang, Nat. Commun., 2012, 3, 1247.

5 C. Dorrer and J. Rühe, Langmuir, 2008, 24, 6154-6158.

6 H. G. Andrews, E. A. Eccles, W. C. E. Schofield and J. P. S. Badyal, Langmuir, 2011, 27, 3798-3802.

7 P. Forbes, Sci. Am., 2008, 299, 88-95.

8 L. Zhai, M. C. Berg, F. Ç. Cebeci, Y. Kim, J. M. Milwid, M. F. Rubner and R. E. Cohen, Nano Lett., 2006, 6, 12131217.

9 S. C. Thickett, C. Neto and A. T. Harris, Adv. Mater., 2011, 23, 3718-3722.

10 B. White, A. Sarkar and A.-M. Kietzig, Appl. Surf. Sci., 2013, 284, 826-836.

11 H. Bai, L. Wang, J. Ju, R. Sun, Y. Zheng and L. Jiang, Adv. Mater., 2014, 26, 5025-5030.

12 D. Zahner, J. Abagat, F. Svec, J. M. J. Fréchet and P. A. Levkin, Adv. Mater., 2011, 23, 3030-3034.

13 I. You, S. M. Kang, S. Lee, Y. O. Cho, J. B. Kim, S. B. Lee, Y. S. Nam and H. Lee, Angew. Chem., Int. Ed., 2012, 51, 6126-6130.

14 S. Nishimoto, A. Kubo, K. Nohara, X. Zhang, N. Taneichi, T. Okui, Z. Liu, K. Nakata, H. Sakai, T. Murakami, M. Abe, T. Komine and A. Fujishima, Appl. Surf. Sci., 2009, 255, 6221-6225.

15 L. Zhang, J. Wu, M. N. Hedhili, X. Yang and P. Wang, J. Mater. Chem. A, 2015, 3, 2844-2852.

16 B.-J. de Gans and U. S. Schubert, Langmuir, 2004, 20, 77897793.

17 B. J. de Gans, P. C. Duineveld and U. S. Schubert, Adv. Mater., 2004, 16, 203-213.

18 P. Gandhidasan and H. I. Abualhamayel, Water Environ. J., 2007, 21, 19-25.

19 L. Zhang, Y. Zhong, D. Cha and P. Wang, Sci. Rep., 2013, 3, 2326.

20 Q. Pan and M. Wang, ACS Appl. Mater. Interfaces, 2009, 1, 420-423.

21 Z. Cheng, M. Du, K. Fu, N. Zhang and K. Sun, ACS Appl. Mater. Interfaces, 2012, 4, 5826-5832.
22 C. Wang, T. Yao, J. Wu, C. Ma, Z. Fan, Z. Wang, Y. Cheng, Q. Lin and B. Yang, ACS Appl. Mater. Interfaces, 2009, 1, 2613-2617.

23 J. R. Dann, J. Colloid Interface Sci., 1970, 32, 302-320.

24 W. Ding, R. Sanchez, M. Ruggles and P. Bernazzani, J. Polym. Res., 2013, 20, 1-8.

25 X. C. Liu, A. Chakraborty and C. Luo, J. Micromech. Microeng., 2010, 20, 095025.

26 X. Jiang, T. Herricks and Y. Xia, Nano Lett., 2002, 2, 13331338.

27 Y. Ofir, B. Samanta, P. Arumugam and V. M. Rotello, Adv. Mater., 2007, 19, 4075-4079.

28 J. Ou, W. Hu, Y. Wang, F. Wang, M. Xue and W. Li, Surf. Interface Anal., 2013, 45, 698-704.

29 L. Zhang, J. Wu, Y. Wang, Y. Long, N. Zhao and J. Xu, J. Am. Chem. Soc., 2012, 134, 9879-9881.

30 B. Wang, J. Li, G. Wang, W. Liang, Y. Zhang, L. Shi, Z. Guo and W. Liu, ACS Appl. Mater. Interfaces, 2013, 5, 1827-1839.

31 F. Mumm, A. T. J. van Helvoort and P. Sikorski, ACS Nano, 2009, 3, 2647-2652.

32 J. C. Love, L. A. Estroff, J. K. Kriebel, R. G. Nuzzo and G. M. Whitesides, Chem. Rev., 2005, 105, 1103-1170.

33 D. Perry and J. A. Taylor, J. Mater. Sci. Lett., 1986, 5, 384-386.

34 J. J. Kim, S.-K. Kim and Y. S. Kim, J. Electroanal. Chem., 2003, 542, 61-66.

35 N. J. Shirtcliffe, G. McHale, M. I. Newton, G. Chabrol and C. C. Perry, Adv. Mater., 2004, 16, 1929-1932.

36 C. R. Crick, J. A. Gibbins and I. P. Parkin, J. Mater. Chem. A, 2013, 1, 5943-5948.

37 W. Shutao, S. Yanlin and J. Lei, Nanotechnology, 2007, 18, 015103.

38 G. Wang and T.-Y. Zhang, ACS Appl. Mater. Interfaces, 2012, 4, 273-279.

39 M. Miwa, A. Nakajima, A. Fujishima, K. Hashimoto and T. Watanabe, Langmuir, 2000, 16, 5754-5760.

40 J. Feng, Z. Qin and S. Yao, Langmuir, 2012, 28, 6067-6075.

41 X. Chen, J. Wu, R. Ma, M. Hua, N. Koratkar, S. Yao and Z. Wang, Adv. Funct. Mater., 2011, 21, 4617-4623.

42 J. Feng, Y. Pang, Z. Qin, R. Ma and S. Yao, ACS Appl. Mater. Interfaces, 2012, 4, 6618-6625.

43 N. Miljkovic, R. Enright, Y. Nam, K. Lopez, N. Dou, J. Sack and E. N. Wang, Nano Lett., 2013, 13, 179-187.

44 K.-C. Park, S. S. Chhatre, S. Srinivasan, R. E. Cohen and G. H. McKinley, Langmuir, 2013, 29, 13269-13277.

45 R. P. Garrod, L. G. Harris, W. C. E. Schofield, J. McGettrick, L. J. Ward, D. O. H. Teare and J. P. S. Badyal, Langmuir, 2006, 23, 689-693.

46 A. Lee, M.-W. Moon, H. Lim, W.-D. Kim and H.-Y. Kim, Langmuir, 2012, 28, 10183-10191. 